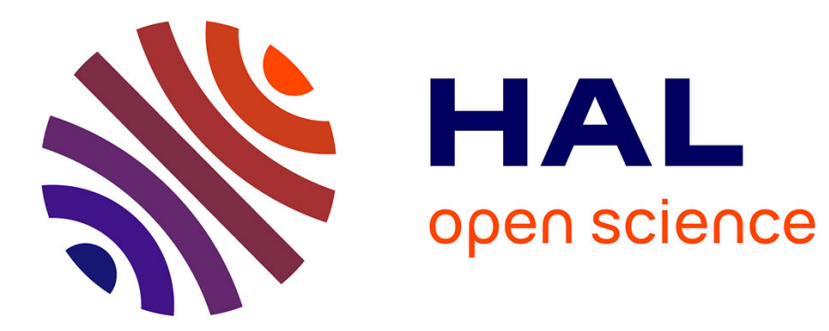

\title{
Optimized PatchMatch for Near Real Time and Accurate Label Fusion
}

Vinh-Thong Ta, Rémi Giraud, D. Louis Collins, Pierrick Coupé

\section{To cite this version:}

Vinh-Thong Ta, Rémi Giraud, D. Louis Collins, Pierrick Coupé. Optimized PatchMatch for Near Real Time and Accurate Label Fusion. MICCAI 2014, Sep 2014, United States. 8 p. hal-01006329

\section{HAL Id: hal-01006329 \\ https://hal.science/hal-01006329}

Submitted on 15 Jun 2014

HAL is a multi-disciplinary open access archive for the deposit and dissemination of scientific research documents, whether they are published or not. The documents may come from teaching and research institutions in France or abroad, or from public or private research centers.
L'archive ouverte pluridisciplinaire HAL, est destinée au dépôt et à la diffusion de documents scientifiques de niveau recherche, publiés ou non, émanant des établissements d'enseignement et de recherche français ou étrangers, des laboratoires publics ou privés. 


\title{
Optimized PatchMatch for Near Real Time and Accurate Label Fusion
}

\author{
Vinh-Thong Ta ${ }^{1,2,3}$, Rémi Giraud ${ }^{1,2,3}$, D. Louis Collins ${ }^{4}$, and Pierrick Coupé ${ }^{1,2}$ \\ 1 Univ. Bordeaux, LaBRI, UMR 5800, PICTURA, F-33400 Talence, France. \\ 2 CNRS, LaBRI, UMR 5800, PICTURA, F-33400 Talence, France. \\ 3 IPB, LaBRI, UMR 5800, PICTURA, F-33600 Pessac, France. \\ 4 McConnell Brain Imaging Centre, Montreal Neurological Institute, McGill \\ University, Montreal, Canada.
}

\begin{abstract}
Automatic segmentation methods are important tools for quantitative analysis of Magnetic Resonance Images. Recently, patchbased label fusion approaches demonstrated state-of-the-art segmentation accuracy. In this paper, we introduce a new patch-based method using the PatchMatch algorithm to perform segmentation of anatomical structures. Based on an Optimized PAtchMatch Label fusion (OPAL) strategy, the proposed method provides competitive segmentation accuracy in near real time. During our validation on hippocampus segmentation of 80 healthy subjects, OPAL was compared to several state-of-theart methods. Results show that OPAL obtained the highest median Dice coefficient $(89.3 \%)$ in less than 1 sec per subject. These results highlight the excellent performance of OPAL in terms of computation time and segmentation accuracy compared to recently published methods.
\end{abstract}

Keywords: PatchMatch, Patch-based Segmentation, Hippocampus

\section{Introduction}

Automatic segmentation methods are efficient tools to produce accurate and reliable measurement dedicated to quantitative analysis of Magnetic Resonance Images (MRI). Over the past years, several paradigms were proposed to achieve the challenging task of brain labeling. First, atlas-based methods involving nonlinear registration of a labeled atlas to the subject to be segmented were proposed [1]. Then, multi-templates warping techniques based on training library of manually labeled templates were introduced. Such methods fuse several similar training templates to achieve better segmentation [2-4]. Multi-templates matching approaches demonstrated competitive segmentation accuracies at the expense an important computational load resulting from multiple nonlinear registrations (i.e., up to several hours). Recently, a nonlocal patch-based label fusion (PBL) strategy [5] has been proposed. Requiring only linear registration, PBL involves patch comparison where the weight assigns to each label depends on the similarity between the current patch and the training patch. The search of similar training patches is based on nonlocal strategy to better handle the inter-subject 
variability and to capture registration inaccuracies. In a limited computational time (i.e., several minutes), this method achieves state-of-the-art segmentation accuracy. Consequently, since its introduction, PBL is intensively studied and many improvements have been proposed [6-9].

Despite these improvements, PBL still suffers from several limitations. First, the search for similar patches is computationally expensive. Although templates preselection [5], patches preselection [5,6] or multiscale strategies [7] have been proposed, an important amount of computation remains dedicated to find similar patches in the training library. Second, the use of preselection strategy can prevent finding the most similar patches. In fact, similar patches can be found in dissimilar training templates. By removing a priori relevant parts of the training library, these preselection approaches can lead to sub-optimal results. Third, in PBL a weight is assigned to a large number of training patches including dissimilar patches. Therefore, resources are uselessly dedicated to estimate negligible weights. Even worse, these dissimilar patches can decrease the segmentation accuracy [8]. Sparsity-based methods can limit this aspect at the expense of an important computational burden $[8,9]$. These limitations may result in suboptimal segmentations and make the current implementations computationally expensive.

In this paper, we introduce a new PBL method based on the PatchMatch (PM) algorithm [10] to address these limitations. Originally, the PM algorithm was introduced to efficiently find an approximate nearest neighbor (ANN) for all patch correspondences between two 2D images. This method is based on a cooperative and randomized strategy resulting in very low computational burden that enables real time image processing. Recently, PM has been used for superresolution of cardiac MRI [11]. Here, we propose a new Optimized PAtchMatch Label fusion (OPAL) method for anatomical structures segmentation by extending the PM approach. Compared to previous PBL methods, OPAL produces segmentations in near real time thanks to the use of the PM scheme. Moreover, OPAL does not require any pre-selection since the search of the most similar patch is achieved over the entire training library leading to higher segmentation accuracy. Finally, by using a very low number of highly similar patches, OPAL limits the introduction of dissimilar patches during label fusion.

The main contributions of this work are: (1) Adaptation of the PM algorithm to label fusion for anatomical structure segmentation in 3D MRI. (2) Acceleration techniques including constrained initialization, parallel processing and optimized distance computation. (3) Validation of OPAL on hippocampus segmentation. (4) Comparison with several state-of-the-art results in terms of computational time and segmentation accuracy.

\section{Methods and Materials}

\subsection{The PatchMatch Algorithm}

The original PM algorithm [10] is a fast and efficient approach that computes patch correspondences between two 2D images (denoted $A$ and $B$ ). The key 
point of this method is that good matches can be propagated to the adjacent patches within an image. This method is based on three steps: initialization, propagation and random search steps. The initialization consists in randomly associating a neighbor for each patch in $A$ with a patch in $B$ to obtain an initial ANN field. The propagation step tries to improve the patch correspondences using the observation that when a patch located at $p=(x, y) \in A$ matches well with a patch located at $q=\left(x^{\prime}, y^{\prime}\right) \in B$ then the adjacent patches of $p \in A$ should match well with the adjacent patches of $q \in B$. The random search step consists in a random sampling around the current ANN to escape from local minima. These two later steps are performed iteratively in order to improve the patch correspondences.

\subsection{Optimized PatchMatch Algorithm}

In contrast to [10] where two 2D images are considered, OPAL finds the patch correspondences between a 3D image $S$ and a library of $3 \mathrm{D}$ templates $L=$ $\left\{T_{1}, \ldots, T_{n}\right\}$ where $n$ is the number of training templates. One advantage of the $\mathrm{PM}$ approach is that the complexity of this algorithm only depends on the size of image $A$ and not on the size of the compared image $B$ (i.e., $L$ in the OPAL case). This important fact allows OPAL to consider the entire image library $L$ without any template preselection step at constant complexity. Moreover, for each patch in $S$, OPAL computes not only one match as done in [10] but the best $k$-ANN matches in $L$.

OPAL is explained in detail below while Figure 1 proposes its schematic overview. For the sake of clarity, only three templates are used in this figure and 3D MRI volumes are displayed in 2D instead of 3D.

Constrained Initialization. In [10], the initialization consists in affecting for each patch located at $(x, y) \in A$ a random correspondence located at $\left(x^{\prime}, y^{\prime}\right) \in B$. In the $3 \mathrm{D}$ case, the natural extension of this step would be to assign for each patch located at $(x, y, z) \in S$ a random patch correspondence located at $\left\{\left(x^{\prime}, y^{\prime}, z^{\prime}\right), t\right\}$ where $t \in\{1, \ldots, n\}$ is the index of the template $T_{t}$ within the library $L$. However, we can take advantage that all MRI volumes in $L$ are linearly registered. Consequently, we propose to constrain the random initial position $\left(x^{\prime}, y^{\prime}, z^{\prime}\right)$ to be within a fixed search window centered around the current position $(x, y, z)$. Then, for each patch in $S$ the index template $t$ is assigned using i.i.d. random variable within $\{1, \ldots, n\}$. Figure 1 (a) shows an illustration of this step, where for each patch in $S$ (only three are displayed) the fixed search window for the random initialization is depicted in dotted lines in different training templates.

As in the PatchMatch algorithm, after this constrained initialization, propagation and random search steps are performed iteratively in order to improve the patch correspondence. Figure 1 also illustrates this iterative process.

Propagation Step with Fast Distance Computation. The OPAL propagation step extends the one proposed by [10] for the 3D case. For each patch located at $(x, y, z) \in S$, we try to improve its ANN by testing if the shifted ANN of its 6 adjacent patches located at $(x \pm 1, y, z),(x, y \pm 1, z)$ and $(x, y, z \pm 1)$ provides a better match. Figures $1(\mathrm{~b})$ and $1(\mathrm{~d})$ illustrate this step, where the blue 


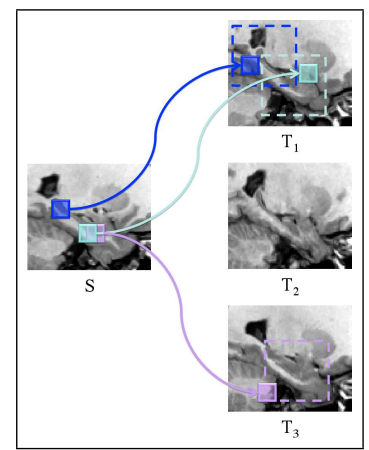

(a) CI

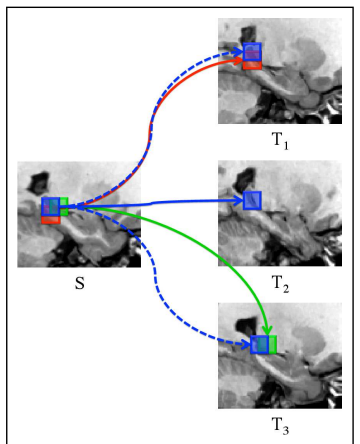

(d) PS for iteration \#2

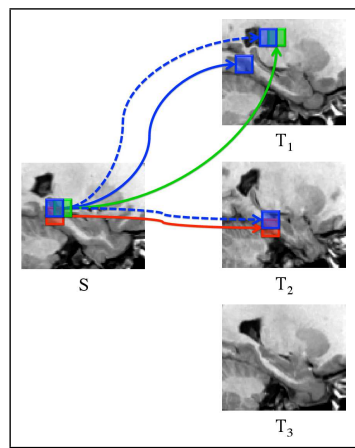

(b) PS for iteration \#1

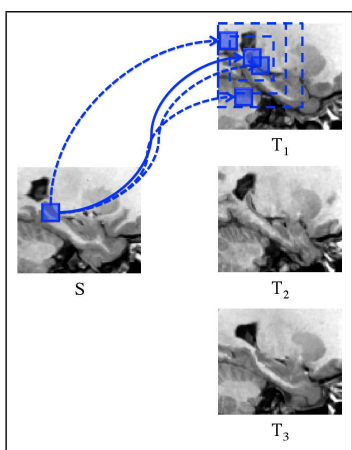

(e) CRS for iteration \#2

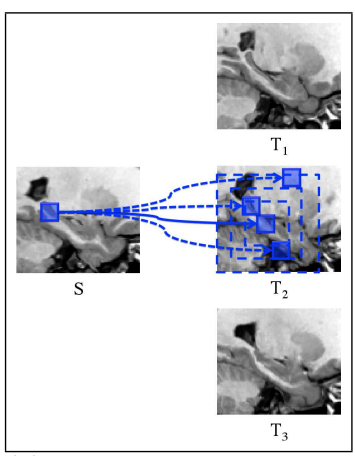

(c) CRS for iteration \#1

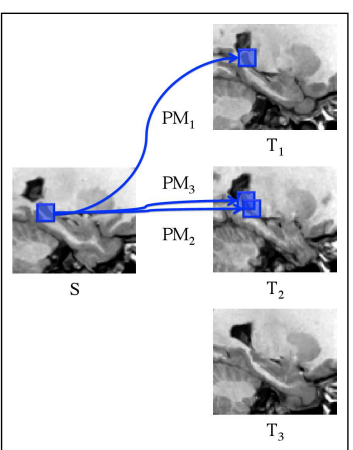

(f) multiple PM

Fig. 1. OPAL main steps. (a) Constrained initialization (CI), (b) and (d) propagation step (PS) for iteration \#1 and \#2, respectively (c) and (e) constrained random search (CRS) for iteration \#1 and \#2, respectively and (f) multiple PM. See text for more details.

dotted lines correspond to the test shifted adjacent neighbors in $L$ in order to improve the current blue patch correspondence. In this example, the best match for the blue patch moves from template $T_{1}$ to $T_{2}$ with iteration \#1 and from $T_{2}$ to $T_{1}$ with iteration \#2. The propagation step is a core stage since it allows a patch correspondence to move over all the templates in $L$. Indeed, since the ANN of the adjacent voxels are not necessarily in the same template, the ANN of the current voxel can move from one template to another one.

Moreover, we propose an acceleration technique based on the observation that the ANN of the adjacent patches are known. Indeed, instead of computing the entire distance (the sum of the squared difference: SSD) between these patches, we take benefit from the patch overlapping by using a sliced SSD where only the non overlapping coordinates are considered. Finally, during the SSD computation, we test if the current sum is superior to the previous minimal SSD. By this way, the SSD estimation can be stopped avoiding extra computation.

Constrained Random Search. In contrast to [10], OPAL deals with a library of images. Therefore, we modify the random search step in order to take into 
account this aspect. Indeed, if we use the original PM algorithm, the random search step should be performed on all the $L$ dimensions, i.e., $x, y, z$ and $t$. However, to ensure spatial consistency, OPAL performs the random search only in the current template that provides the current best patch correspondence (i.e., $t$ is fixed, and we random on $\left.\left(x^{\prime}, y^{\prime}, z^{\prime}\right) \in T_{t}\right)$ within a decaying search window as in [11]. Figures 1(c) and 1(e) present examples of such fixed template random search where the decaying search windows are represented in dotted blue lines.

Multiple PM and Parallel Computation. Finally, while in [10] only the best match is estimated, OPAL computes $k$-ANN matches in $L$ to perform label fusion. In the literature, an extension of the original PM algorithm to $k$-ANN case was proposed [12]. The suggested strategy is to build a stack of the best visited matches to obtain the $k$-ANN. However, to parallelize such an approach, the current image $S$ must be split into several parts with problems of patches boundaries overlapping between threads. Therefore, in OPAL, we decided to based the $k$-ANN search on independent $k$-PM enabling a more efficient and simple multi-threading. Figure 1(f) illustrates the result of the multiple PM step where here $k=3$ and, each $\mathrm{PM}_{i=1,2,3}$ denotes an individual PM.

Patch-based Label Fusion. At the end the process, the $k$-ANN are estimated for all the patches in $S$. Thus, the location and the SSD between the patches of $S$ and their $k$-ANN in $L$ are known. Therefore, to obtain the final segmentation, we used the PBL method presented in [5]. However, in OPAL only the $k$ most similar patches are used (limiting segmentation error) and the entire library is considered (increasing segmentation accuracy). When the same ANN is selected several times it will be considered several times during label fusion. Finally, to further improve segmentation quality, label fusion is performed over the whole patch as done in $[6,9]$ and not only using the central voxel.

\subsection{Validation}

Dataset. The proposed method was evaluated on the International Consortium for Brain Mapping (ICBM) dataset. Part of this dataset consists of $80 \mathrm{MR}$ images of young and healthy individuals with manual segmentations following the Pruessner's protocol [13]. The MRI scans were acquired with a 1.5T Philips GyroScan imaging system $(1 \mathrm{~mm}$ thick slices, $\mathrm{TR}=17 \mathrm{~ms}$, TE $=10 \mathrm{~ms}$, flip angle $=30^{\circ}, 256 \mathrm{~mm}$ field of view). The estimated intra-class reliability coefficient was of 0.90 for inter- ( 4 raters) and 0.92 for intra-rater ( 5 repeats) reliability.

Preprocessing. All the images were preprocessed through the following pipeline: estimation of the standard deviation of noise [14]; denoising using the optimized nonlocal means filter [15]; correction of inhomogeneities using N3 [16]; registration to stereotaxic space based on a linear transform to the ICBM152 template $\left(1 \times 1 \times 1 \mathrm{~mm}^{3}\right.$ voxel size) [17]; linear intensity normalization of each subject on template intensity; brain extraction using BEaST [7]; image cropping around the structures of interest; and cross-normalization of the MRI intensity between the subjects within the estimated brain mask with [18].

Quality Metric and Compared Methods. To validate the proposed method a leave-one-out cross validation procedure was used. During our validation, 
we investigated the impact of the patch size and of the number of neighbors (i.e., number of PM). Moreover, OPAL was compared with Atlas-Based Method (ABM) [1], Multi-Templates Matching (MTM) [4], Patch-Based Label fusion (PBL) [5], Sparse Representation Classification (SRC) [8], Discriminative Dictionary Learning for Segmentation (DDLS) and Fixed Discriminative Dictionary Learning for Segmentation (F-DDLS) [8] since all these methods were validated on the same dataset. The segmentation quality was estimated with the Dice coefficient by comparing the expert-based segmentations with the automatic segmentations. The median Dice coefficients and computational times presented in Table 1 are the published values. These values include segmentation of both, left and right hippocampus. OPAL was implemented in MATLAB using multithreaded C-MEX code. Our experiments were carried out using a server of 16 cores at $2.6 \mathrm{GHz}$ with $100 \mathrm{~GB}$ of RAM. The number of threads was equal to $k$ and the number of inner iterations of OPAL was set to 5 as in [10].

\section{$3 \quad$ Results}

Influence of Parameters. Figure 2 shows the influence of the number of neighbors and of the patch size on the segmentation quality and on the computational time. Similarly to previous PBL methods $[5,8]$, we found that patches of size $5 \times 5 \times 5$ and $7 \times 7 \times 7$ voxels provides the best results with a slight advantage for patches of size $5 \times 5 \times 5$ voxels $(89.4 \%$ for $k=20)$. Moreover, we found that the median Dice coefficient reached a plateau around 10-ANN. Interestingly, this number is in line with the suggested number of templates in multi-templates matching methods [4]. As expected, bigger patches and larger number of ANN required higher computational time. Consequently, our experiments suggest that using patch size of $5 \times 5 \times 5$ and $k=10$ offers a good trade off between segmentation accuracy $(89.3 \%)$ and computational time $(0.89 \mathrm{~s})$.
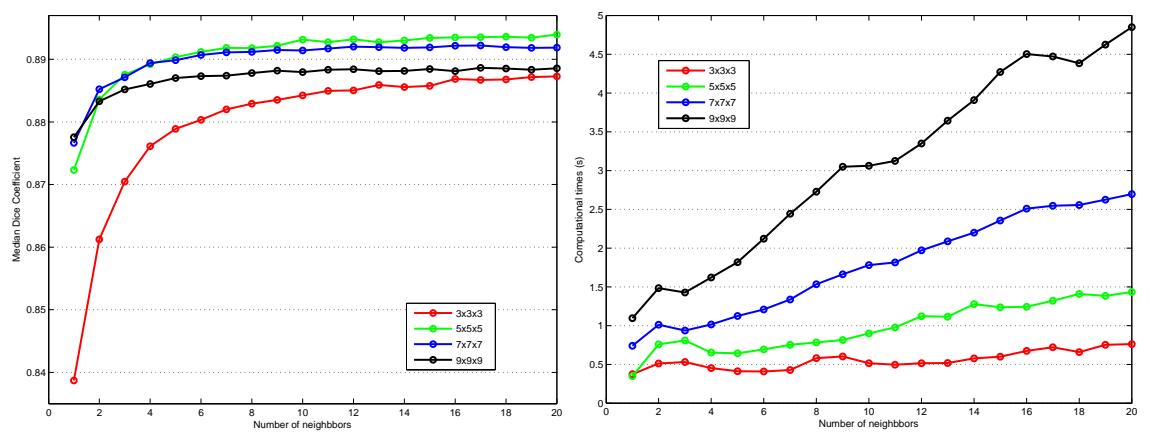

Fig. 2. Median Dice coefficient according to the patch size and the number of neighbors (at left) and the corresponding computational time (at right). 
Table 1. Methods comparison in terms of segmentation accuracy and computational time on the ICBM dataset.

\begin{tabular}{lcc}
\hline Method & Median Dice Coefficient & Computational Time by Subject \\
\hline ABM [1] & $86.4 \%$ & $358 s$ \\
PBL [5] & $88.2 \%$ & $662 \mathrm{~s}$ \\
MTM [4] & $88.6 \%$ & $3974 \mathrm{~s}$ \\
F-DDLS [8] & $88.6 \%$ & $193 \mathrm{~s}$ \\
SRC [8] & $88.7 \%$ & $5587 \mathrm{~s}$ \\
DDLS [8] & $89.0 \%$ & $943 \mathrm{~s}$ \\
OPAL & $\mathbf{8 9 . 3} \%$ & $\mathbf{0 . 8 9 s}$ \\
\hline
\end{tabular}

Comparison with State-of-the-Art Methods. The comparison of OPAL performance with 6 other methods is presented in Table 1. The presented values are the results published by the authors for the segmentation of both hippocampi on the ICBM dataset. The provided computational times do not include template preselection while only OPAL does not require it. Therefore, the computational times are under-estimated except for OPAL. Moreover, for the F-DDLS an offline training step of $1781 \mathrm{~s}$ is needed. However, OPAL obtained the highest median Dice coefficient in the fastest manner. These results highlights the excellent performance of OPAL in terms of both: segmentation accuracy and computational time. Compared to the original PBL [5], OPAL obtained better accuracy $700 \times$ faster. Moreover, OPAL obtained the highest Dice coefficient for a computational time $200 \times$ faster than the fastest published method on the used dataset (F-DDLS [8]). Finally, compared to the most accurate method (DDLS [8]), OPAL obtained higher Dice coefficient for a computational time $1000 \times$ faster.

\section{Conclusion}

In this paper, we propose a novel patch-based segmentation method based on an optimized PatchMatch label fusion. The OPAL method enables high quality segmentation in near real time. Experiments show that the proposed method obtained competitive results compared to the state-of-the-art approaches. Indeed, OPAL obtained the highest median Dice coefficient in a much faster manner. In addition, the near real time capabilities of OPAL pave the way for new applications for label fusion segmentation. For instance, OPAL can be used as an efficient automatic or interactive segmentation tool in medical visualization software. Finally, as future work, OPAL will be validated on multi-sites datasets containing pathological cases and extended to multi-label segmentation.

\section{Acknowledgments}

This study has been carried out with financial support from the French State, managed by the French National Research Agency (ANR) in the frame of the Investments for the future Programme IdEx Bordeaux (ANR-10-IDEX-03-02), 
Cluster of excellence CPU and TRAIL (HR-DTI ANR-10-LABX-57). We also acknowledge funding from the Fonds de Recherche Québec - Santé (FRQS-Pfizer).

\section{References}

1. Collins, D.L., et al: Automatic 3-D model-based neuroanatomical segmentation. Human Brain Mapping 3(3) (1995) 190-208

2. Heckemann, R.A., et al: Automatic anatomical brain MRI segmentation combining label propagation and decision fusion. NeuroImage 33(1) (2006) 115-126

3. Lötjönen, J.M., et al: Fast and robust multi-atlas segmentation of brain magnetic resonance images. NeuroImage 49(3) (2010) 2352-2365

4. Collins, D.L., Pruessner, J.C.: Towards accurate, automatic segmentation of the hippocampus and amygdala from mri by augmenting animal with a template library and label fusion. Neuroimage 52(4) (2010) 1355-1366

5. Coupé, P., et al: Patch-based segmentation using expert priors: Application to hippocampus and ventricle segmentation. NeuroImage 54(2) (2011) 940-954

6. Rousseau, F., et al: A supervised patch-based approach for human brain labeling. Medical Imaging, IEEE Transactions on 30(10) (2011) 1852-1862

7. Eskildsen, S.F., et al: BEaST: Brain extraction based on nonlocal segmentation technique. NeuroImage 59(3) (2012) 2362-2373

8. Tong, T., et al: Segmentation of MR images via discriminative dictionary learning and sparse coding: Application to hippocampus labeling. NeuroImage 76 (2013) $11-23$

9. Wu, G., et al: A generative probability model of joint label fusion for multi-atlas based brain segmentation. Medical image analysis (2013)

10. Barnes, C., et al: Patchmatch: A randomized correspondence algorithm for structural image editing. In: ACM SIGGRAPH 2009 Papers. (2009) 24:1-24:11

11. Shi, W., et al: Cardiac image super-resolution with global correspondence using multi-atlas patchmatch. In Mori, K., Sakuma, I., Sato, Y., Barillot, C., Navab, N., eds.: MICCAI 2013. Volume 8151 of LNCS. Springer Berlin Heidelberg (2013) 9-16

12. Barnes, C., et al: The generalized patchmatch correspondence algorithm. In Daniilidis, K., Maragos, P., Paragios, N., eds.: ECCV 2010. Volume 6313 of LNCS. Springer Berlin Heidelberg (2010) 29-43

13. Pruessner, J., et al: Volumetry of hippocampus and amygdala with high-resolution MRI and three-dimensional analysis software: minimizing the discrepancies between laboratories. Cerebral cortex 10(4) (2000) 433-442

14. Coupé, P., et al: Robust Rician noise estimation for MR images. Medical image analysis 14(4) (2010) 483-493

15. Coupé, P., et al: An optimized blockwise nonlocal means denoising filter for 3-D magnetic resonance images. Medical Imaging, IEEE Transactions on 27(4) (2008) $425-441$

16. Sled, J.G., et al: A nonparametric method for automatic correction of intensity nonuniformity in MRI data. Medical Imaging, IEEE Transactions on 17(1) (1998) $87-97$

17. Collins, D.L., et al: Automatic 3D intersubject registration of MR volumetric data in standardized talairach space. Journal of computer assisted tomography 18(2) (1994) 192-205

18. Manjón, J.V., et al: Robust MRI brain tissue parameter estimation by multistage outlier rejection. Magnetic Resonance in Medicine 59(4) (2008) 866-873 